\title{
Prostatakarzinom: Protonen kaum weniger toxisch als IMRT
}

Weil sich mit der Protonentherapie Tumoren gezielter bestrahlen lassen, ist das Interesse daran auch für das Prostatakarzinom groß. In den USA haben Radiologen jetzt geprüft, ob sie weniger Nebenwirkungen hat als die intensitätsmodulierte Radiotherapie (IMRT).

W ie in Deutschland wird die Protonentherapie in den USA im Vergleich zur etablierten IMRT bisher nur an wenigen Zentren angeboten. Aus früheren US-Studien gibt es Hinweise, dass es mit der IMRT weniger gastrointestinale Toxizitäten gibt als mit der Protonentherapie. Allerdings sind die Daten nicht für die ganzen USA repräsentativ.

Um den Langzeiteffekt der beiden Therapieformen bei Männern mit einem noch nicht metastasierten Prostatakarzinom zu ermitteln, werteten jetzt USRadiologen um James B. Yu Daten aller Medicare-Patienten älter als 65 Jahre im Zeitraum zwischen 2008 und 2009 aus.

Von 27.647 Männern mit Prostatakarzinom erhielten 553 der Männer eine
Protonentherapie (2\%) und 27.100 eine IMRT (98\%). Auffallend waren toxische Wirkungen vor allem im Urogenitalbereich. Ihr Anteil lag zwar sechs Monate nach Therapie bei Männern mit Protonentherapie signifikant niedriger als bei Männern mit IMRT ( 5,9 vs. 9,5\%, p $=0,03)$. Doch nach einem Jahr war der Unterschied nicht mehr signifikant $(\mathrm{p}=$ $0,66)$. Die Unterschiede bei gastrointestinalen Toxizitäten waren über den gesamten Studienzeitraum nicht signifikant.

Die Radiologen weisen aber darauf hin, dass ihnen Informationen über die Strahlendosis für die Auswertung fehlten. Möglicherweise sei die Dosierung bei der IMRT höher gewesen, was die höhere Toxizität sechs Monate nach Stu- dienbeginn erklären könnte. Eventuell könnte die Ursache aber auch darin liegen, dass die Bestrahlung per IMRT eher regional als bei der Protonentherapie ist, die gezielt die Prostata im Fokus hat. Solange es keine prospektiven Vergleichsstudien gebe, sei es nicht gerechtfertigt, die Protonentherapie flächendeckend anzubieten, so die amerikanischen Kollegen.

Fazit: Die Toxizität nach Protonentherapie bei Männern mit nicht metastasiertem Prostatakarzinom ist zumindest in dieser US-amerikanischen Studie nur unwesentlich niedriger als nach IMRT. Noch gibt es aber zu wenig Daten aus randomisierten Vergleichsstudien, um einen klaren klinischen Vorteil der Protonentherapie zu belegen.

Peter Leiner

Yu JB et al. Proton Versus Intensity-Modulated Radiotherapy for Prostate Cancer: Patterns of Care and Early Toxicity. J Natl Cancer Inst. 2013;105(1):25-32.

\section{Progression nach Docetaxel-Therapie des Prostatakarzinoms: Neue Hoffnung}

\section{Die Zwischenanalyse der Phase-III-Studie COU-AA-301 hatte gute Ergebnisse für Abirateron bei metastasiertem kastrationsresistentem Prostatakarzinom geliefert. Die endgültige Auswertung der Studie bestätigt nun eine signifikante Verlängerung des Gesamtüberlebens.}

\footnotetext{
A birateron ist ein selektiver Inhibitor der Androgen-Biosynthese und aktiv gegen metastasierte kastrationsresistente Prostatakarzinome mit oder ohne vorheriger Chemotherapie. In der doppelblinden, placebokontrollierten Phase-III-Studie wurde die Substanz an 1195 Patienten mit metastasiertem kastratationsresistentem Prostatakarzinom und Progression unter Docetaxel untersucht. Im Verhältnis 2:1 erhielten die Patienten entweder Abirateron (1.000 mg, 1-mal täglich, oral) plus Prednison (5 mg, 2-mal täglich, oral) oder Placebo plus Prednison. Die Verblindung wurde aufgehoben, weil in der Zwischenauswertung nach median 12,8 Monaten Abirateron das Gesamtüberleben von 10,9 Monaten unter Prednison allein auf 14,8 Monate verbessert hatte.
}

Die Patienten der Placebogruppe konnten nun auf Abirateron umgestellt werden.

Die endgültigen Ergebnisse der Studie bis zum Zeitpunkt der Entblindung bestätigen die Resultate der Zwischenauswertung. Nach median 20,2 Monaten erreichten die Patienten unter Abirateron ein signifikant längeres medianes Gesamtüberleben (primärer Endpunkt) (15,8 vs. 11,2 Monate; Hazard Ratio [HR] 0,74; $\mathrm{p}<0,0001)$. Die Überlegenheit war in allen Subgruppen nachweisbar, unabhängig vom ECOG-Performance-Status, von Schmerzen zu Studienbeginn, der Anzahl der bisherigen Chemotherapien, der Art der Progression, der vorherigen Docetaxel-Anwendung, dem Grund des Absetzens von Docetaxel, des Therapiestarts mit Abirateron und der Dauer der
Docetaxel-Therapie, auch wenn die Ergebnisse aufgrund der geringen Patientenzahl nicht in allen Subgruppenanalysen signifikant waren. Auch hinsichtlich der medianen Dauer bis zur PSA-Progression (8,5 vs. 6,6 Monate; HR 0;63; $\mathrm{p}<$ $0,0001)$, des medianen radiologischen progressionsfreien Überlebens (5, 6 vs. 3,6 Monate; HR 0,66; $\mathrm{p}<0,0001)$ und des Anteils der Patienten mit PSA-Response ( 29,5 vs $5,5 \%$; $\mathrm{p}<0,0001)$ war Abirateron signifikant besser als Placebo.

Bezüglich unerwünschter Ereignisse der Grade 3-4 gab es keine Unterschiede zwischen beiden Armen.

Fazit: Diese Endauswertung bestätigt, dass Abirateron das Gesamtüberleben von Patienten mit metastasiertem kastrationsresistentem, nach Docetaxel progredientem Prostatakarzinom signifikant verlängert.

Judith Neumaier

Fizazi K et al. Abiraterone acetate for treatment of metastatic castration-resistant prostate cancer: final overall survival analysis of the COU-AA-301 randomised, double-blind, placebo-controlled phase 3 study. Lancet Oncol. 2012;13(10):983-92. 